\title{
Staufen blocks autophagy in neurodegeneration
}

Sharan Paul ${ }^{1}$, Warunee Dansithong ${ }^{1}$, Mandi Gandelman ${ }^{1}$, Tao $\mathrm{Zu}^{2}$, Laura P.W. Ranum², Karla

P. Figueroa ${ }^{1}$, Daniel R. Scoles ${ }^{1}$, and Stefan M. Pulst ${ }^{1 \#}$

${ }^{1}$ Department of Neurology, University of Utah, 175 North Medical Drive East, 5th Floor, Salt

Lake City, Utah, 84132. ${ }^{2}$ Department of Neurology, University of Florida, Gainesville, FL 32610, USA

\#Author for correspondence: stefan.pulst@hsc.utah.edu

\section{Abstract}

Response to cellular stress represents a highly conserved pathway in evolution. Cells respond to stress with modified synthesis of new proteins by the formation of stress granules (SGs), inhibition of translation initiation and by increased recycling of cellular components through autophagy. One of the master regulators of this response is the mechanistic target of rapamycin (mTOR) kinase ${ }^{1,2}$. We recently reported that Staufen1 (STAU1), a stress granule protein, was overabundant in the rare neurodegenerative disorder SCA2 and provided a link between SG formation and autophagy ${ }^{3}$. In cells harboring mutant ATXN2, STAU1 could also be increased by bafilomycin A consistent with impaired autophagosome lysosome fusion ${ }^{3}$. Here we now examine this association and show the molecular mechanism leading to autophagic block in cells with microtubule associated protein tau (MAPT), presenilin 1 (PSEN1), huntingtin (HTT), TAR DNA-binding protein-43 gene (TARDBP) or C9orf72-SMCR8 complex subunit (C9orf72) mutations underlying a great number of neurodegenerative diseases ${ }^{4-8}$. We found that STAU1 overabundance was present in all cell lines and animal models tested, that it was posttranslational, and that it was associated with an increase in phosphorylated mTOR (P-mTOR) and autophagic block. Exogenous expression of STAU1 in wild-type cells was sufficient to 
reduce autophagic flux by itself. Mechanistically, STAU1 directly interacted with the $m T O R$ 5'UTR and enhanced mTOR translation. As STAU1 itself is degraded by autophagy, this interaction and the resulting autophagic block results in a maladaptive amplifying response to chronic stress. Targeting STAU1 by RNAi decreased mTOR hyperactivity and normalized mTOR downstream targets in dividing cells, post-mitotic neurons and animal models of SCA2 and ALS-TDP-43 or C9orf72 associated neurodegeneration. In summary, STAU1 is necessary and sufficient to mediate a maladaptive cellular stress response and is a novel target for RNAimediated treatment of neurodegenerative diseases.

\section{Introduction}

Staufen was identified in the fly as a protein determining the spatiotemporal localization of maternal mRNAs in oocytes and eggs ${ }^{9-11}$. Mammals have two Staufen proteins (STAU1 and STAU2), both involved in mRNA localization and transport ${ }^{11-16}$. Binding of STAU1 to the 3'UTRs of specific classes of RNAs leads to Staufen-mediated decay (SMD) whereas binding to the 5'UTR results in enhanced translation ${ }^{16-19}$.

We previously showed that STAU1 was part of the ATXN2 protein complex and that fibroblasts (FBs) from individuals with SCA2 or TDP-43 ${ }^{\mathrm{G} 298 \mathrm{~S}}$ mutations had elevated STAU1 levels ${ }^{3}$. When STAU1 expression was reduced by genetic interaction, motor behavior of SCA2 mice improved with normalization of key Purkinje cell proteins and reduction of cytoplasmic aggregates containing ATXN2 (ref. 3). We found that a large number of differentially regulated genes in our SCA2 model overlapped with RNAs identified by crosslinking with STAU1(ref. 20) and demonstrated a direct interaction of the PCP2-3'UTR with STAU1 (ref. 3).

\section{Results and Discussion}

\section{STAU1 overabundance is common in cell lines derived from patients with}

\section{neurodegenerative diseases}


We now explored whether STAU1 overabundance was represented a more generalized response of cells stressed by presence of mutant disease proteins or other chronic stressors. We therefore obtained FBs from ALS patients with a variety of TDP-43 mutations or with repeat expansion in C9orf72, HD patients with huntingtin (HTT) poly-Q expansion and Alzheimer's disease patients with PSEN1 or MAPT mutations. By quantitative western blot analysis, STAU1 was significantly increased in all patient cell lines (Fig. 1a-e). The STAU1 mRNA levels, however, were unchanged (Extended Data Fig. 1a,b).

\section{Stau1 overabundance is associated with autophagy dysfunction}

In prior studies, we had found that STAU1 was primarily degraded through the autophagosome, presumably owing to its association with RNA granules. We therefore tested whether autophagy was impaired in cells with endogenously elevated STAU1 and whether exogenous expression of mutant disease-linked proteins could result in STAU1 overabundance and impaired autophagy. A master regulator of autophagy is $\mathrm{mTOR}$ and once phosphorylated (P-mTOR), it serves as a kinase of several targets including ribosomal protein S6 kinase (S6K1), and eukaryotic initiation factor 4E-binding protein (4E-BP1). FBs obtained from SCA2 patients all showed an increase in total mTOR and active P-mTOR levels, and an increase in p62 and LC3-II levels indicating reduced autophagic flux (Fig. 1e). Activation of mTOR was also evident in cells from patients with ALS, HD and AD (Fig. 1a-d). Increased STAU1 and mTOR levels in these FBs were not the result of transcriptional changes (Extended Data Fig. 1a,b).

It is possible that patient-derived cells may contain other unknown mutations in addition to the respective causative mendelian mutation. We therefore used two independent approaches to establish a causative relationship from disease proteins to STAU1 overabundance to autophagosome dysfunction. First, we modified HEK-293 cells using the CRISPR/Cas9 system to introduce disease-causing mutations. When compared to wild-type 
HEK-293 cells, cells with endogenous mutated ATXN2, designated ATXN2-Q22/58 KI (ref. 3) showed increased STAU1, mTOR and P-mTOR levels. As expected in the presence of overabundant P-mTOR, downstream effectors of mTOR signaling showed increased abundance and phosphorylation as well (P-S6 and P-4E-BP1) (Extended Data Fig. 2a).

Second, we expressed disease causing proteins exogenously in HEK-293 cells to determine whether they recapitulated STAU1 overabundance and mTOR activation. This included wild-type or mutant TDP-43 (G298S, A382T and G348C), C-terminal fragment (CTF) of TDP-43 and MAPT mutation 4,7,21. Exogenous expression of disease-linked proteins resulted in increased mTOR activities (Extended Data Fig. 2b-d). These results support that presence of mutant disease-linked proteins are directly related to STAU1 overabundance and autophagy impairment.

\section{Stau1 is overabundant in mouse models of neurodegeneration}

Although STAU1 overabundance and impaired autophagy were seen in a number of cell lines from patients with various neurodegenerative conditions, this analysis used cells that are actively dividing. To examine STAU1 levels in post-mitotic neurons in vivo we analyzed brain protein extracts from a number of mouse models expressing human mutant proteins. As previously reported, STAU1 is overabundant in cerebellar extracts of SCA2 mice $3,22,23$. We now examined the state of autophagy in these mice and find that the in vivo results mirrored the ones obtained using SCA2 cell lines (Fig. 2a).

We then extended this analysis to a mouse line expressing wild-type TDP-43 (refs. 24-26) and a mouse line expressing a human BAC with mutant C9orf72 repeat ${ }^{27}$. By western blot analysis, both TDP-43 ${ }^{T g /+}$ hemizygous and BAC-C9orf72 mice demonstrated greatly increased Stau1 levels similar to those seen in $A T X N 2^{Q 127}$ mice (Fig. 2b-d). Associated with elevated Stau1, we found that autophagy pathway components were altered predicting reduced flux by inefficient autophagosome-lysosome fusion. This included total and phosphorylated mTor as well as p62 
and LC3-II which functions as a selective autophagy receptor for degradation of ubiquitinated substrates (Fig. 2a-d).

\section{STAU1 directly interacts with the 5'UTR of $m$ TOR and enhances its translation}

We determined if exogenous expression of STAU1 in HEK-293 cells resulted in the same alterations that were seen in cells with neurodegenerative disease-linked mutations. Expression of STAU1 resulted in increased mTOR, P-mTOR, p62 and LC3-II levels (Fig. 3a). Levels of mTOR transcripts were unchanged (Fig. 3b). These results indicated that STAU1 overabundance was sufficient to induce autophagic dysfunction.

As STAU1 can enhance translation of mRNAs upon binding to 5'UTRs (ref. 17), we tested whether STAU1 was able to bind mTOR RNA and whether this interaction would increase translation of $m T O R$ mRNA. We used two independent approaches to establish an interaction between STAU1 and mTOR RNA. First, Protein-RNA immunoprecipitation (non-RNase A treated) followed by RT-PCR in HEK-293 cells expressing Flag-tagged STAU1 demonstrated that Flag-STAU1 pulled down mTOR mRNA but not control GAPDH (Fig. 3c). Second, using bacterially expressed recombinant His-tagged STAU1 protein and in vitro transcribed DIGlabelled mTOR RNA probes, we were able to map the interaction of STAU1 to the mTOR-5'UTR by northwestern blot analysis (Fig. 3d,e).

To test the consequence of STAU1 binding to the mTOR RNA, we inserted the mTOR-5'UTR upstream of luciferase. In the presence of exogenous STAU1, translation was greatly increased (Fig. 3f-h). We tested for the specificity of STAU1 interaction with the UTR sequence by substituting the mTOR-5'UTR with the PCP2-5'UTR as PCP2 is known only to be subjected to SMD not mediated by its 5'UTR (ref. 3). Exogenous STAU1 resulted in significant induction of luciferase activity only from mTOR-5'UTR-LUC construct (Fig. 3f-h). The double stranded RNA binding domain 3 (dsRBD3) of STAU1 acts as the major functional domain for target RNA interactions ${ }^{28-30}$. The translational increase was dependent on the presence of this domain as its 
deletion abrogated the effect of STAU1 on translation (Fig. 3i-k). These data explain mTOR increase and map the interaction to the 5'UTR of the mTOR RNA and the dsRBD3 domain of STAU1.

\section{STAU1 responds to a variety of stressors}

Accumulating evidence indicates that stress-induced intracellular changes lead to neurotoxicity in a number of neurodegenerative diseases ${ }^{31-34}$. We wanted to test whether subjecting cells to various forms of stress other than expression of disease-linked genes would affect STAU1 and autophagic function. We used HEK-293 cells and subjected them to endoplasmic reticulum (ER) stressors (thapsigargin or tunicamycin), a calcium ionophore (ionomycin), oxidative stress (tunicamycin), metal stressor (sodium arsenite) and temperature stress (hyperthermia) followed by western blotting. The different forms of stress all induced STAU1 overabundance, mTOR elevation and increased phosphorylation of mTOR targets (Fig 4a-e). To confirm the involvement of STAU1 in the activation of this pathway we established primary cultures of cortical neurons from wild-type and Stau1/-- mice. For wild-type neurons, treatments with thapsigargin induced Stau1 overabundance and mTor activation with increased p62 and LC3-II levels. In contrast, Stau1/-- neurons showed minimal response to thapsigargin (Fig. 4f), indicating that the activation of mTOR and downstream targets is STAU1 dependent. Although the precise signaling pathways are not yet known, these findings indicate that STAU1 is positioned at the crossroads of several cellular stress pathways.

\section{Reduction of overabundant STAU1 normalizes autophagy}

Exogenous STAU1 expression was sufficient to change autophagic flux, shown above. We now needed to determine whether STAU1 was necessary. To test this, we knocked STAU1 down in the presence of mutant disease proteins in vitro and in vivo. When we depleted STAU1 in ATXN2-Q58 KI cells by STAU1 RNAi, mTOR levels were normalized. Reduced mTOR activity was verified by reduced P-mTOR, P-S6 and P-4EBP1 levels, and restoration of p62 and LC3-II 
turnover (Fig. 5a). Thus, reducing STAU1 levels was sufficient in rescuing autophagic flux in SCA2 cells.

We next proceeded to determine if lowering STAU1 levels could restore autophagic flux in ALS cellular models. Like in ATXN2-KI cells, lowering STAU1 levels by RNAi in ALS-TDP-43 or C9orf72 cell models also restored autophagic flux (Fig. 5b,c). Of note, silencing of STAU1 had no effect on TDP-43 protein levels. These data indicate that STAU1 knockdown may be useful for preventing maladaptive responses to cellular stress.

Since lowering STAU1 levels can normalize aberrant autophagy associated with SCA2, TDP-43 and C9orf72 mutations, we wanted to test if reducing STAU1 levels is protective of pathological stress. We treated HEK-293 cells with STAU1 RNAi followed by wild-type or mutant TDP-43 transfection. In all cases, lowering STAU1 normalized mTOR signaling and autophagy (Extended Data Fig. 3). These results establish that STAU1 plays a key role in protecting cells from a wide variety of pathological stressors.

\section{In vivo results}

The availability of a mouse with a Stau1 loss-of-function allele allowed us to determine the effect of Stau1 reduction using genetic interaction. We crossed SCA2 or TDP-43 mouse lines with Stau1 KO mice generating animals that had $50 \%$ or $0 \%$ Stau1 protein levels $3,22,24,35$. We had previously observed that reduction of Stau1 in vivo improved motor behavior and proteomics in SCA2 mice ${ }^{3}$. We then measured mTor, P-mTor, p62, and LC3-II to determine autophagic flux. Changing Stau1 dosage in wild-type animals did not alter autophagy marker proteins in an appreciable way. In contrast, reducing pathologically elevated Stau1 in SCA2 mice improved markers of autophagy in a dose-dependent fashion such that levels of all proteins were returned to near normal (Fig. 6a,b,d). Similar to in SCA2 mice, reducing Stau1 dosage by $50 \%$ in TDP- $43^{T g /+}$ mice resulted in decreased mTOR activity and restoration of autophagy pathway proteins (Fig. 6c,e). 
Our results have implications for the basic understanding of mechanisms regulating autophagy and for identifying novel targets for the treatment of neurodegeneration. Current understanding of autophagy emphasizes regulation by kinase cascades and by transcriptional changes in key genes involved in autophagy ${ }^{36-39}$. We now describe an additional regulatory mechanism that involves RNA-binding proteins, formation of SGs with the ability of acutely influencing the translation of already transcribed mRNAs. The double-stranded RNA-binding protein STAU1 directly interacts with mTOR-mRNA and enhances its translation. As STAU1 is itself degraded by autophagy, this establishes an amplification mechanism for autophagy inhibition.

STAU1 is also a key player in RNA decay, and its overabundance likely affects RNA networks that involve cellular functions and signaling cascades in addition to autophagy. Little is known about these networks in individual cell types other than HEK-293 cells ${ }^{3}$, but it is reasonable to assume that some of the cell-type specificity in neurodegenerative diseases is mediated via RNA decay. It is tempting to speculate that overabundance of STAU1 also provides a bridge from RNA granule formation to aberrant phase transition to hydrogels, a mechanism important in several neurodegenerative diseases ${ }^{40}$.

In summary, we found that STAU1 is highly overabundant in multiple cellular and mouse models of neurodegenerative disease and that targeting STAU1 could restore abnormal downstream signaling to autophagy. STAU1 may be an outstanding novel target for antisense oligonucleotide (ASO)- or RNAi-based therapies. Our past studies in an SCA2 mouse model showed that reducing STAU1 by only $50 \%$ improved motor behavior ${ }^{3}$. We now show that this improvement is associated with restoration of P-mTOR levels and downstream targets of mTOR-kinase in vivo. For C9orf72 neurodegeneration, targeting STAU1 may provide a better or additional target as the C9orf72 gene product appears to have essential cellular functions. As compared with other approaches to improving autophagy, targeting STAU1 may have the added advantage of normalizing SMD as well. 


\section{References}

1. Menzies, F.M. et al. Autophagy and Neurodegeneration: Pathogenic Mechanisms and Therapeutic Opportunities. Neuron. 2017 Mar 8;93(5):1015-1034 (2017)

2. Monahan, Z., Shewmaker, F. \& Pandey, U.B. Stress granules at the intersection of autophagy and ALS. Brain Res. 2016 Oct 15;1649(Pt B):189-200 (2016).

3. Paul, S., Dansithong, W., Figueroa, K.P., Scoles, D.R. \& Pulst, S.M. Staufen1 links RNA stress granules and autophagy in a model of neurodegeneration. Nat Commun. 2018 Sep 7;9(1):3648 (2018).

4. Hoover, B.R. et al. Tau mislocalization to dendritic spines mediates synaptic dysfunction independently of neurodegeneration. Neuron. 2010 Dec 22;68(6):1067-1081 (2010).

5. Karch, C.M. \& Goate, A.M. Alzheimer's disease risk genes and mechanisms of disease pathogenesis. Biol Psychiatry. 2015 Jan 1;77(1):43-51 (2015).

6. Gil, J.M. \& Rego, A.C. Mechanisms of neurodegeneration in Huntington's disease. Eur J Neurosci. 2008 Jun;27(11):2803-20 (2008).

7. Pesiridis, G.S., Lee, V.M. \& Trojanowski, J.Q. Mutations in TDP-43 link glycine rich domain functions to amyotrophic lateral sclerosis. Hum Mol Genet. Oct 15;18(R2): R156-162 (2009).

8. Haeusler, A.R., Donnelly, C.J. \& Rothstein, J.D. The expanding biology of the C9orf72 nucleotide repeat expansion in neurodegenerative disease. Nat Rev Neurosci. 2016 Jun;17(6):383-395 (2016).

9. Broadus, J., Fuerstenberg, S. \& Doe, C.Q. Staufen-dependent localization of prospero mRNA contributes to neuroblast daughter-cell fate. Nature 391: 792-795 (1998).

10. Roegiers, F. \& Jan, Y.N. Staufen: a common component of mRNA transport in oocytes and neurons? Trends Cell Biol. Jun;10(6): 220-224 (2000).

11. Heraud-Farlow, J.E. \& Kiebler, M.A. The multifunctional Staufen proteins: conserved roles from neurogenesis to synaptic plasticity. Trends Neurosci. 2014 Sep;37(9):470-479 (2014).

12. Kiebler, M.A. et al. The mammalian staufen protein localizes to the somatodendritic domain of cultured hippocampal neurons: implications for its involvement in mRNA transport. J Neurosci. 1999 Jan 1;19(1):288-297 (1999).

13. Tang, S. J., Meulemans, D., Vazquez, L., Colaco, N. \& Schuman, E. A role for a rat homolog of Staufen in the transport of RNA to neuronal dendrites. Neuron 32, 463-475 (2001).

14. Miki, T., Takano, K., Yoneda, Y. The role of mammalian Staufen on mRNA traffic: A view from its nucleocytoplasmic shuttling function. Cell Struct Funct 30: 51-56 (2005). 
15. Maquat, L.E. \& Gong, C. Gene expression networks: competing mRNA decay pathways in mammalian cells. Biochem Soc Trans. 2009 Dec;37(Pt 6):1287-1292. (2009).

16. Park, E. \& Maquat, L.E. Staufen-mediated mRNA decay. Wiley Interdiscip Rev RNA 4: 423-435 (2013).

17. Dugré-Brisson, S. et al. Interaction of Staufen1 with the 5' end of mRNA facilitates translation of these RNAs. Nucleic Acids Res 33: 4797-4812 (2005).

18. Kim, Y.K., Furic, L., Desgroseillers, L. \& Maquat, L.E. Mammalian Staufen1 recruits Upf1 to specific mRNA 3'UTRs so as to elicit mRNA decay. Cell 2005 120: 195-208 (2005).

19. Kim, Y.K. et al. Staufen1 regulates diverse classes of mammalian transcripts. EMBO J 26: 2670-2681 (2007).

20. Sugimoto, Y. et al. hiCLIP reveals the invivo atlas of mRNA secondary structures recognized by Staufen1. Nature 519: 491-494 (2015).

21. Igaz, L.M. et al. Expression of TDP-43 C-terminal Fragments in Vitro Recapitulates Pathological Features of TDP-43 Proteinopathies. J Biol Chem. 2009 Mar 27;284(13):8516-8524 (2009).

22. Hansen, S.T., Meera, P., Otis, T.S. \& Pulst, S.M. Changes in Purkinje cell firing and gene expression precede behavioral pathology in a mouse model of SCA2. Hum Mol Genet 22: 271-283 (2013).

23. Dansithong, W. et al. Ataxin-2 regulates RGS8 translation in a new BAC-SCA2 transgenic mouse model. PLoS Genet 11: e1005182 (2015).

24. Wils, H. et al. TDP-43 transgenic mice develop spastic paralysis and neuronal inclusions characteristic of ALS and frontotemporal lobar degeneration. Proc Natl Acad Sci U S A. 2010 Feb 23;107(8):3858-3863 (2010).

25. Janssens, J. et al. Overexpression of ALS-associated p.M337V human TDP-43 in mice worsens disease features compared to wild-type human TDP-43 mice. Mol Neurobiol. 2013 Aug;48(1):22-35 (2013).

26. Becker, L.A. et al. Therapeutic reduction of ataxin-2 extends lifespan and reduces pathology in TDP-43 mice. Nature. 2017 Apr 20;544(7650):367-371 (2017).

27. Liu, Y. et al. C9orf72 BAC Mouse Model with Motor Deficits and Neurodegenerative Features of ALS/FTD. Neuron. 2016 May 4;90(3): 521-534 (2016).

28. Wickham, L., Duchaîne, T., Luo, M., Nabi, I.R. \& DesGroseillers, L. Mammalian staufen is a double-stranded-RNA- and tubulin-binding protein which localizes to the rough endoplasmic reticulum. Mol Cell Biol 19: 2220-2230 (1999).

29. Duchaîne, T.F. et al. Staufen2 isoforms localize to the somatodendritic domain of neurons and interact with different organelles. J Cell Sci. 2002 Aug 15;115(Pt 16):328595 (2002). 
30. Ravel-Chapuis, A. et al. The RNA-binding protein Staufen1 is increased in DM1 skeletal muscle and promotes alternativepre-mRNA splicing. J Cell Biol. 2012 Mar 19;196(6):699-712 (2012).

31. Lindholm, D., Wootz, H. \& Korhonen, L. ER stress and neurodegenerative diseases. Cell Death Differ. 2006 Mar;13(3):385-392 (2006).

32. Chen, L. \& Liu, B. Relationships between Stress Granules, Oxidative Stress, and Neurodegenerative Diseases. Oxid Med Cell Longev. 2017;2017: 1809592 (2017).

33. Liu, Z., Zhou, T., Ziegler, A.C., Dimitrion, P. \& Zuo L. Oxidative Stress in Neurodegenerative Diseases: From Molecular Mechanisms to Clinical Applications. Oxid Med Cell Longev. 2017;2017: 2525967 (2017).

34. Chen, P., Miah, M.R. \& Aschner M. Metals and Neurodegeneration [version 1; referees: 3 approved] F1000Research 2016, 5 (F1000 Faculty Rev): 366 (2016).

35. Vessey, J.P. et al. A loss of function allele for murine Staufen1 leads to impairment of dendritic Staufen1-RNP delivery and dendritic spine morphogenesis. Proc Natl Acad Sci U S A. 105: 16374-16379 (2008).

36. Fleming, A., Noda, T., Yoshimori, T. \& Rubinsztein, D.C. Chemical modulators of autophagy as biological probes and potential therapeutics. Nat Chem Biol. 2011 Jan;7(1):9-17 (2011).

37. Rahman, M.A. \& Rhim, H. Therapeutic implication of autophagy in neurodegenerative diseases. BMB Rep. 2017 Jul;50(7):345-354 (2017).

38. Guo, F., Liu, X., Cai, H. \& Le, W. Autophagy in neurodegenerative diseases: pathogenesis and therapy. Brain Pathol. 2018 Jan;28(1):3-13 (2018).

39. Giampieri, F. et al. Autophagy in Human Health and Disease: Novel Therapeutic Opportunities. Antioxid Redox Signal. 2019 Feb 1;30(4):577-634 (2019).

40. Guo, Q. et al. In Situ Structure of Neuronal C9orf72 Poly-GA Aggregates Reveals Proteasome Recruitment. Cell. 2018 Feb 8;172(4):696-705.e12 (2018).

\section{Acknowledgments}

We thank Prof. Dr. Michael A. Kiebler, Ludwig Maximilian University of Munich, Germany for providing Stau1 ${ }^{\text {tm1Apa(-/-) }}\left(\right.$ Stau $\left.1^{-/}\right)$mice. We thank Brian S. Marshall for technical assistance and mouse handlings. Funding: This work was supported by grants R01NS097903, RC4NS073009 and R56NS33123 from the National Institutes of Neurological Disorders and Stroke to SMP, and Noorda foundation to SMP, and grants R37NS033123 and R21NS081182 to DRS and SMP. SMP received grant support from the Target ALS Foundation and is a consultant for Ataxion 
Pharmaceuticals and Progenitor Life Sciences. The funders had no role in study design, data collection and analysis, decision to publish, or preparation of the manuscript.

\section{Author contributions}

S.P., W.D. and S.M.P. conceived and designed the experiments. Experiments were performed by S.P., W.D. and M.G. BAC-C9orf72 mouse extracts are provided by T.Z. and L.P.W.R. Data analyses was performed by S.P., W.D., M.G., D.R.S. and S.M.P. All figures were generated by S.P. and W.D. Administrative assistance was provided by K.P.F. The manuscript was written by S.P., W.D., D.R.S. and S.M.P., and critically reviewed by L.P.W.R., D.R.S. and S.M.P.

\section{Competing interests}

The authors declare no competing interests. 


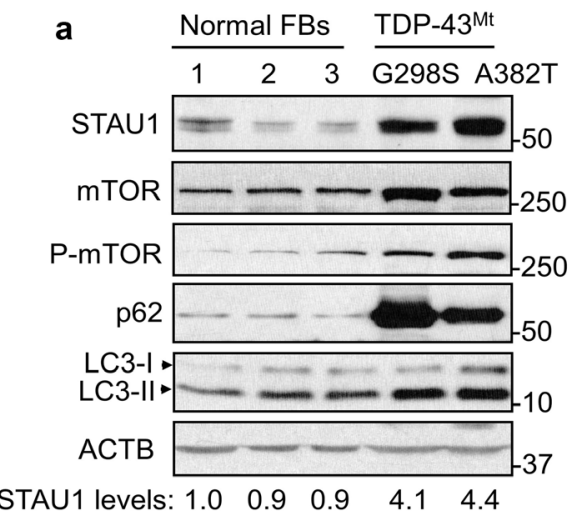

C

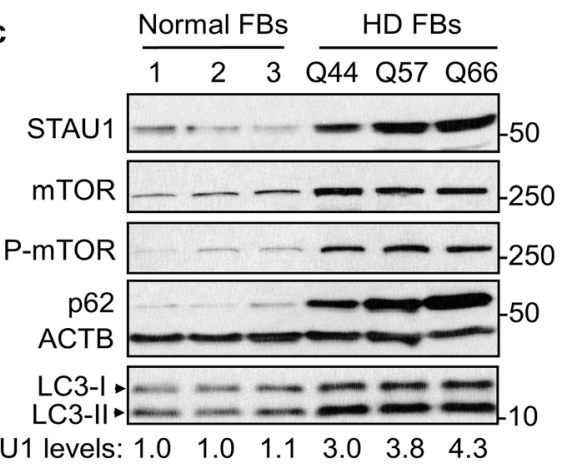

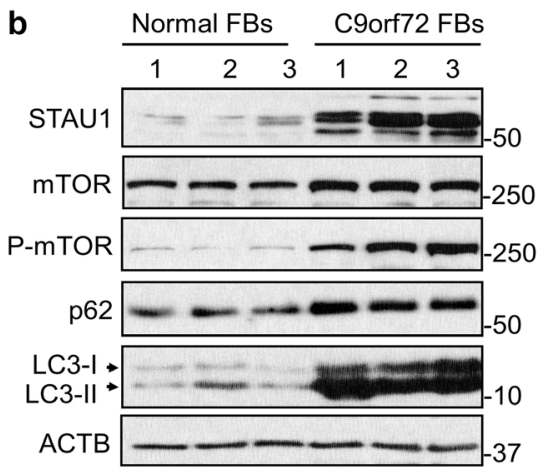

STAU1 levels: $\begin{array}{llllll}1.0 & 0.9 & 1.2 & 4.7 & 7.2 & 6.6\end{array}$

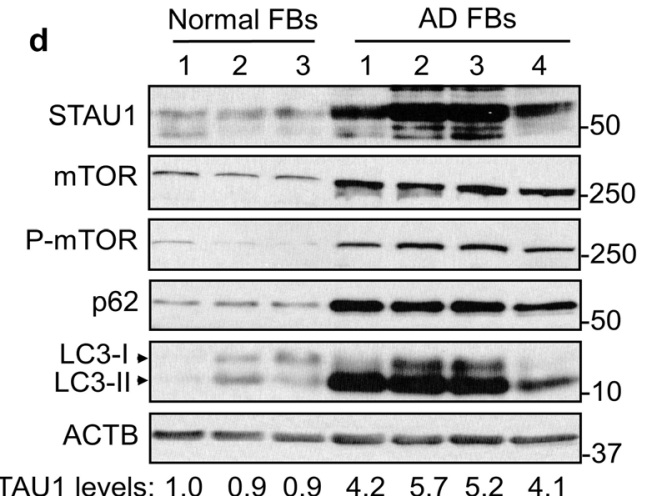

STAU1 levels: $\begin{array}{lllllll}1.0 & 0.9 & 0.9 & 4.2 & 5.7 & 5.2 & 4.1\end{array}$

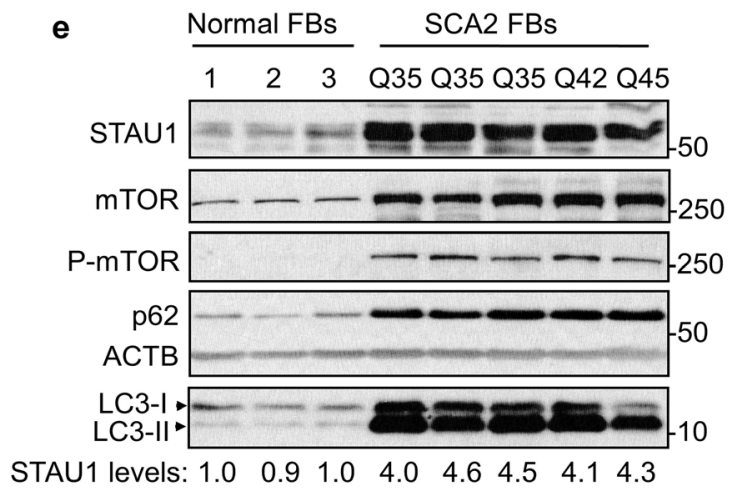

Figure 1 | STAU1 levels and mTOR activity are increased in neurodegenerative states. Western blot analysis of extracts from ALS FBs with TDP-43 (a) or C9orf72 mutations (b), HD FBs (c), AD FBs [(PSEN1 mutations: M146I, E184D and Intron4 Var) and MAPT mutation: (N279K)] (d) and SCA2 FBs (e) show increased STAU1 levels compared with normal controls. All FB extracts show increased mTOR and P-mTOR levels and increased p62 and LC3-II levels compared with control FBs (a-e). $\beta$-Actin was used as loading control and representative blots of three independent experiments are shown 
a

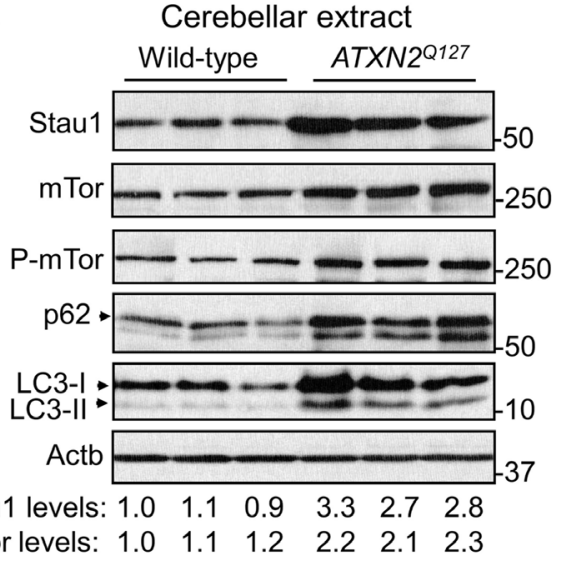

b Cerebellar extract

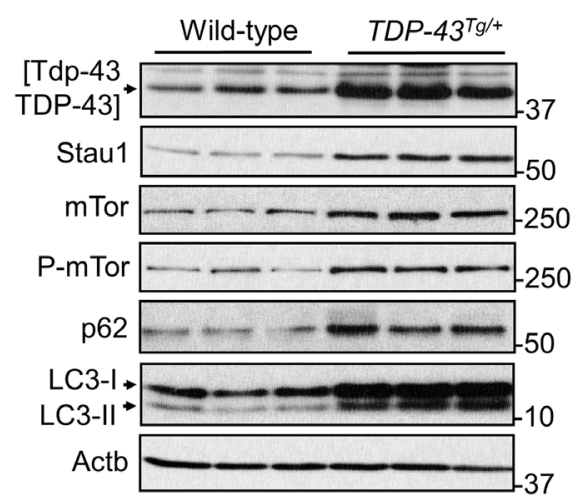

Stau1 levels: $\begin{array}{llllll}1.0 & 1.1 & 1.2 & 2.5 & 2.8 & 3.1\end{array}$

mTor levels: $1.0 \quad 1.0 \quad 0.9 \quad 2.3 \quad 2.4 \quad 2.3$

d

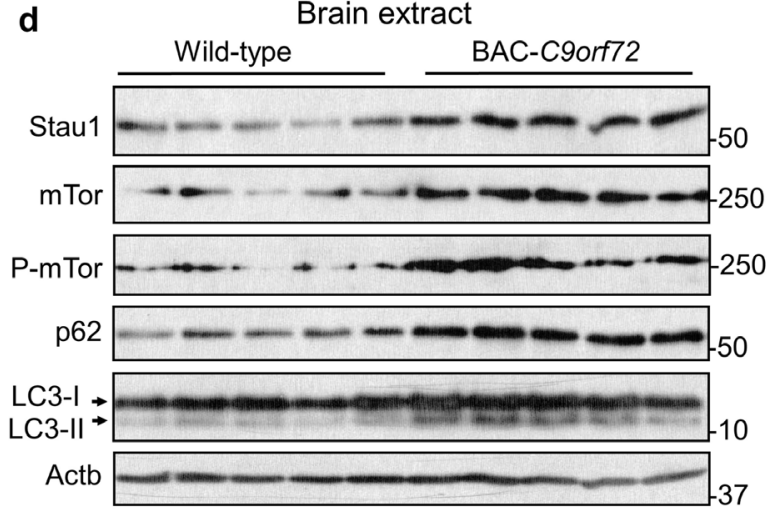

Stau1 levels: $\begin{array}{llllllllll}1.0 & 1.2 & 0.9 & 1.0 & 1.1 & 2.2 & 2.0 & 2.6 & 2.6 & 3.4\end{array}$

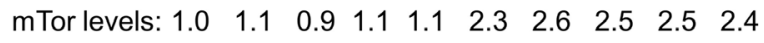

Figure 2 | Stau1 levels and mTOR activity are increased in SCA2 and ALS models.

Western blot analyses of $A T X N 2^{Q 127}$ mouse $^{22}$ (a) cerebellar extracts (24 weeks of age; 3 animals per group), and cerebellar (b) and spinal cord (c) extracts from $T D P-43^{T g /+}$ hemizygous mice $^{24}$ (8 weeks of age; 3 animals per group) show increased Stau1, mTor and P-mTor, p62 and LC3-II levels compared with wild-type litter mate controls. d BAC-C9orf72 mouse ${ }^{27}$ brain extracts (20 weeks of age; 5 animals per group) have increased Stau1, mTor, P-mTor, p62 and LC3-II levels compared with wild-type controls. Each lane represents an individual mouse. $\beta$ Actin was used as a loading control and the blots are from three replicate experiments 

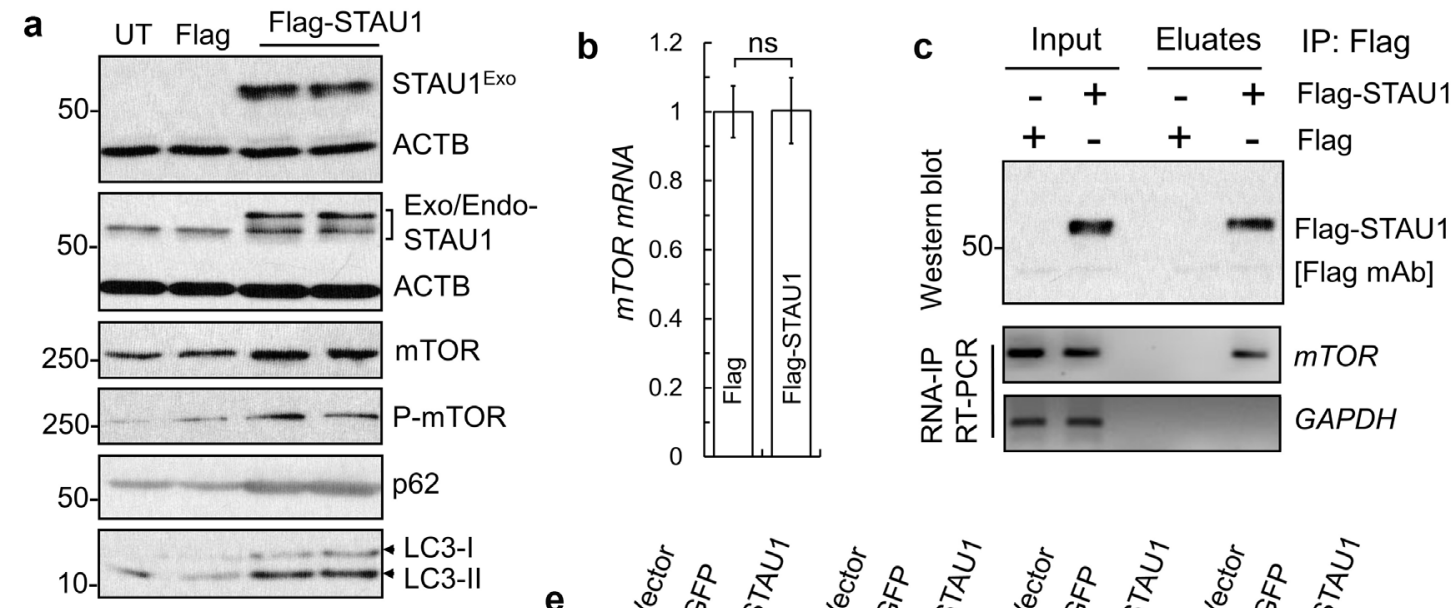
d DIG-mTOR(5'UTR) DIG-mTOR(3'UTR) DIG 9 9 P DIG

f \begin{tabular}{llll} 
CMV & 5'UTR-MTOR LUC \\
\hline CMV & 5'UTR-PCP2 & LUC \\
\hline
\end{tabular}
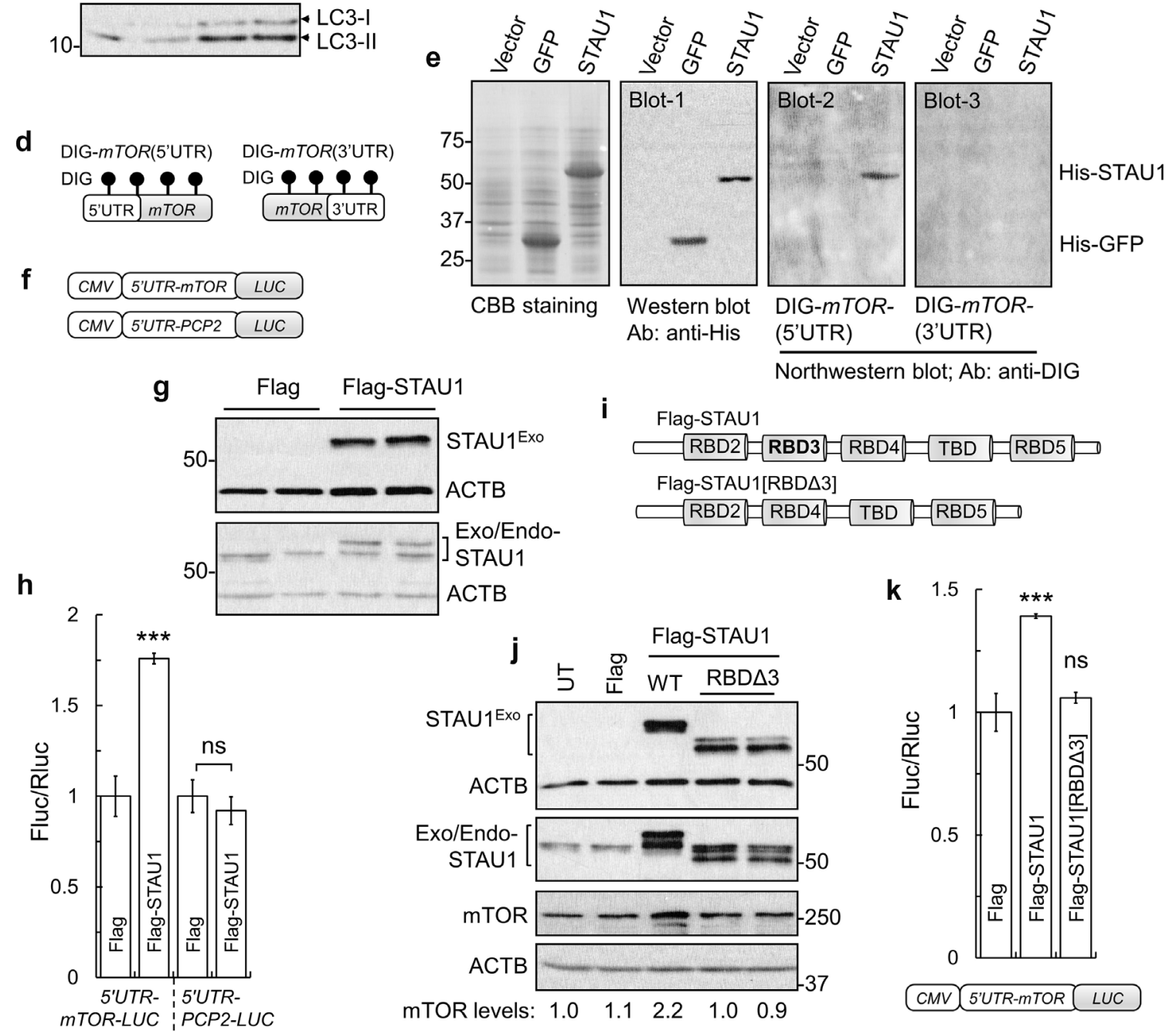

Figure 3 | STAU1 interaction with the 5'UTR of $m$ TOR enhances $m$ TOR translation and mTOR signaling. a, b STAU1 overexpression elevates mTOR. a HEK-293 cells exogenously expressing Flag-tagged STAU1 were analyzed 48 hrs post-transfection by western blotting and 
showed increased mTOR, P-mTOR, p62 and LC3-II levels. b mTOR mRNA levels, however, were unchanged by qRT-PCR analyses. RNA expression levels were normalized to ACTB. $\mathbf{c}-\mathbf{h}$ STAU1 regulates mTOR expression through binding to mTOR-5'UTR. c Non-RNase A treated HEK-293 whole cell extracts expressing Flag or Flag-STAU1 were immunoprecipitated with Flag mAb beads. Flag-STAU1 pulled down mTOR RNA by RT-PCR analysis. d, e STAU1 binds directly to human $m T O R$ RNA in a manner requiring the 5'UTR by Northwestern blot analysis. d Schematic of DIG-labeled human mTOR RNA probes with 5'UTR and 3'UTR sequences. e STAU1 directly interacts with mTOR-5'UTR RNA (blot 2), but not with mTOR-3'UTR RNA (blot 3). Bacterially expressed His-STAU1, His-GFP, or control bacterial lysate were stained with Coomassie brilliant blue (CBB) on SDS-PAGE or western blotted with anti-His antibody (blot 1). Protein blots were hybridized with DIG-labelled $m$ TOR RNA probes followed by anti-DIG antibody staining. His-GFP shows no interactions. f Schematic of mTOR-5'UTR-LUC and PCP2-5'UTR-LUC (control) reporters. Exogenous STAU1 expression induces increased expression of the mTOR-5'UTR-LUC construct but not PCP2-5'UTR-LUC (PCP2-3'UTR substrate for SMD (ref. 3)) on luciferase reporter assay. Western blotting showing expression of STAU1 $(\mathbf{g}, \mathbf{h})$. i-k The RBD3 is required to induce mTOR expression. Schematic representation of STAU1 and mutant STAU1 excluding RBD3 (STAU1[RBDA3]) (i). HEK-293 cells were transfected with STAU1 constructs alone or co-transfected with mTOR-5'UTR-LUC reporter construct followed by western blotting or luciferase assay (j, k). Significantly increased endogenous mTOR level (j) or luciferases activity (k) are achieved only through the STAU1 construct retaining the RBD3. Luciferase expression normalized to Renilla luciferase (RLU) and $\beta$-Actin as a loading control; blots are from three replicate experiments. Data are mean $\pm S D$, ${ }^{* * *} P<0.001$, Student t-test 

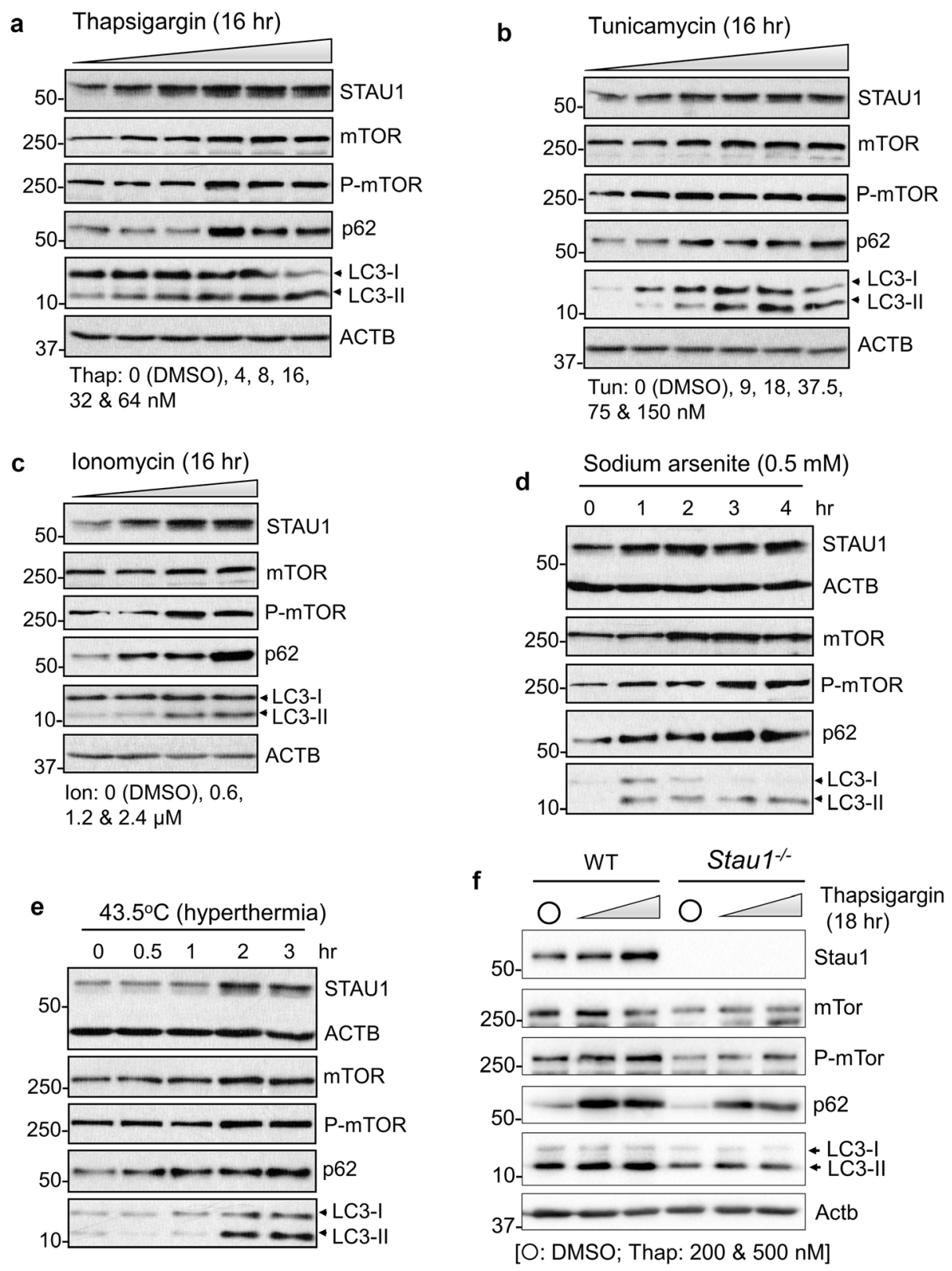

Figure 4 | STAU1 responds to a variety of stressors. a-e HEK-293 cells were treated with the indicated stressors and analyzed by western blotting. The treatments increased STAU1, mTOR and P-mTOR levels, and reduced autophagy activity (increased p62 and LC3-II levels). f Mouse cortical neurons treated with thapsigargin also showed increased Stau1, mTor, P-mTor, p62, and LC3-II (left), but neurons null for Stau1 are resistant to thapsigargin-mediated induction of these genes (right). $\beta$-Actin was used as loading control. Representative blots of three independent experiments are shown 


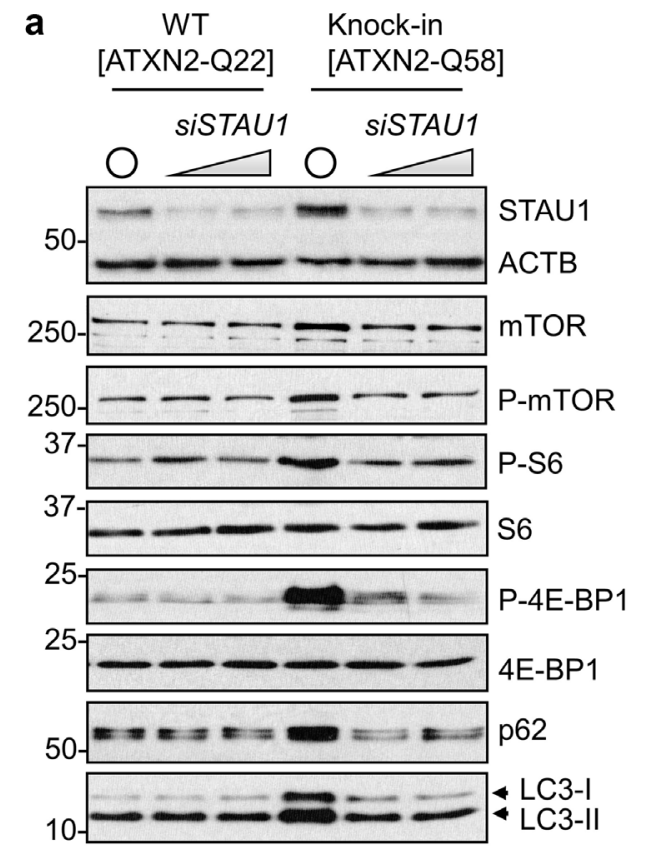

[O: Cont. siRNA $200 \mathrm{nM}$; siSTAU1: 100 and $200 \mathrm{nM}$ ] b
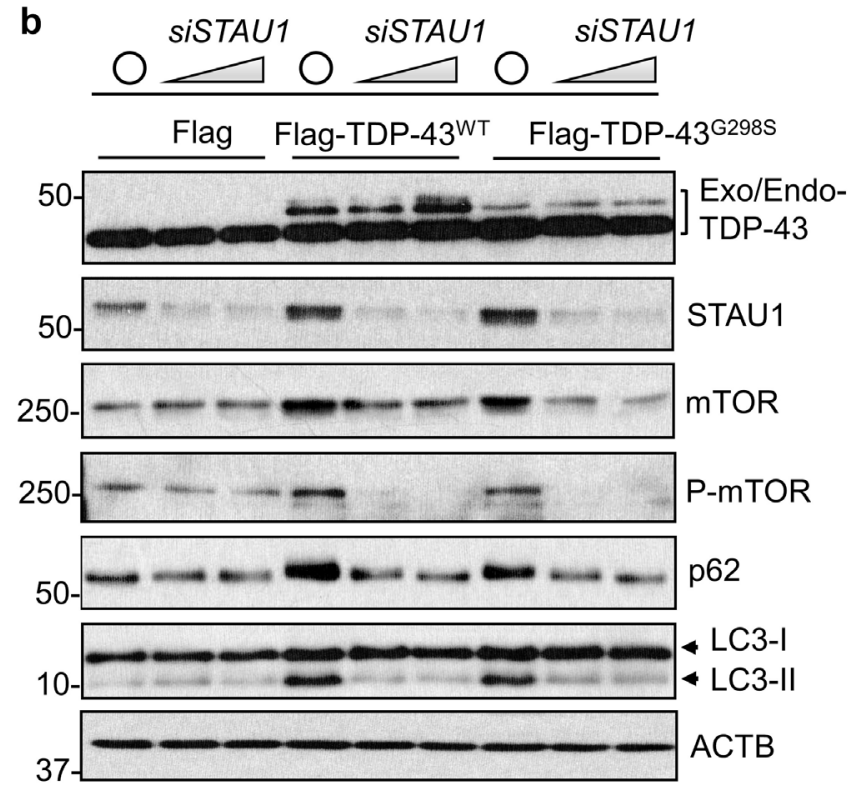

[O: Cont. siRNA $200 \mathrm{nM}$; siSTAU1: $100 \& 200 \mathrm{nM}]$

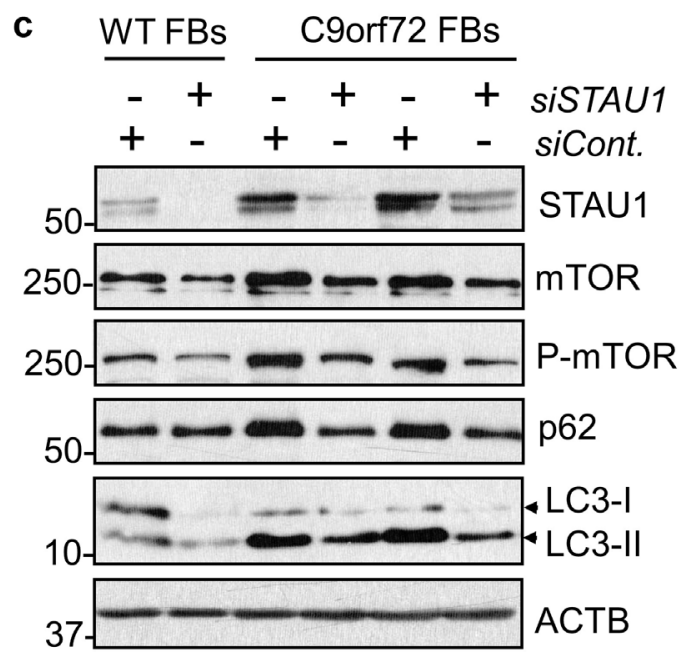

[siCont. \& siSTAU1: $200 \mathrm{nM}$ ]

Figure 5 | Silencing of STAU1 reduces mTOR activation in SCA2 and ALS cellular models. a STAU1 depletion lowers mTOR activity concurrently with mTOR targets and restores autophagic pathway proteins in ATXN2-Q22/58 KI cells. Cells were transfected with STAU1 
siRNA and analyzed by western blotting. STAU1 depletion in ATXN2-Q22/58 KI cells results in decreased mTOR levels, mTOR activity (reduced P-mTOR, P-S6 and P-4E-BP1 levels), as well as p62 and LC3-II levels, indicating restored autophagy activity. b, c STAU1 depletion lowers mTOR levels and restores autophagic pathway proteins in cells with TDP-43 (b) or C9orf72 mutation (c). b Western blots of HEK-293 cells expressing Flag-tagged wild-type or mutant TDP-43 or empty vectors followed by transfection with STAU1 siRNA. c ALS-C9orf72 FBs transfected with STAU1 siRNA and analyzed by western blotting. $\beta$-Actin is used as a loading control and the blots are from three replicate experiments 

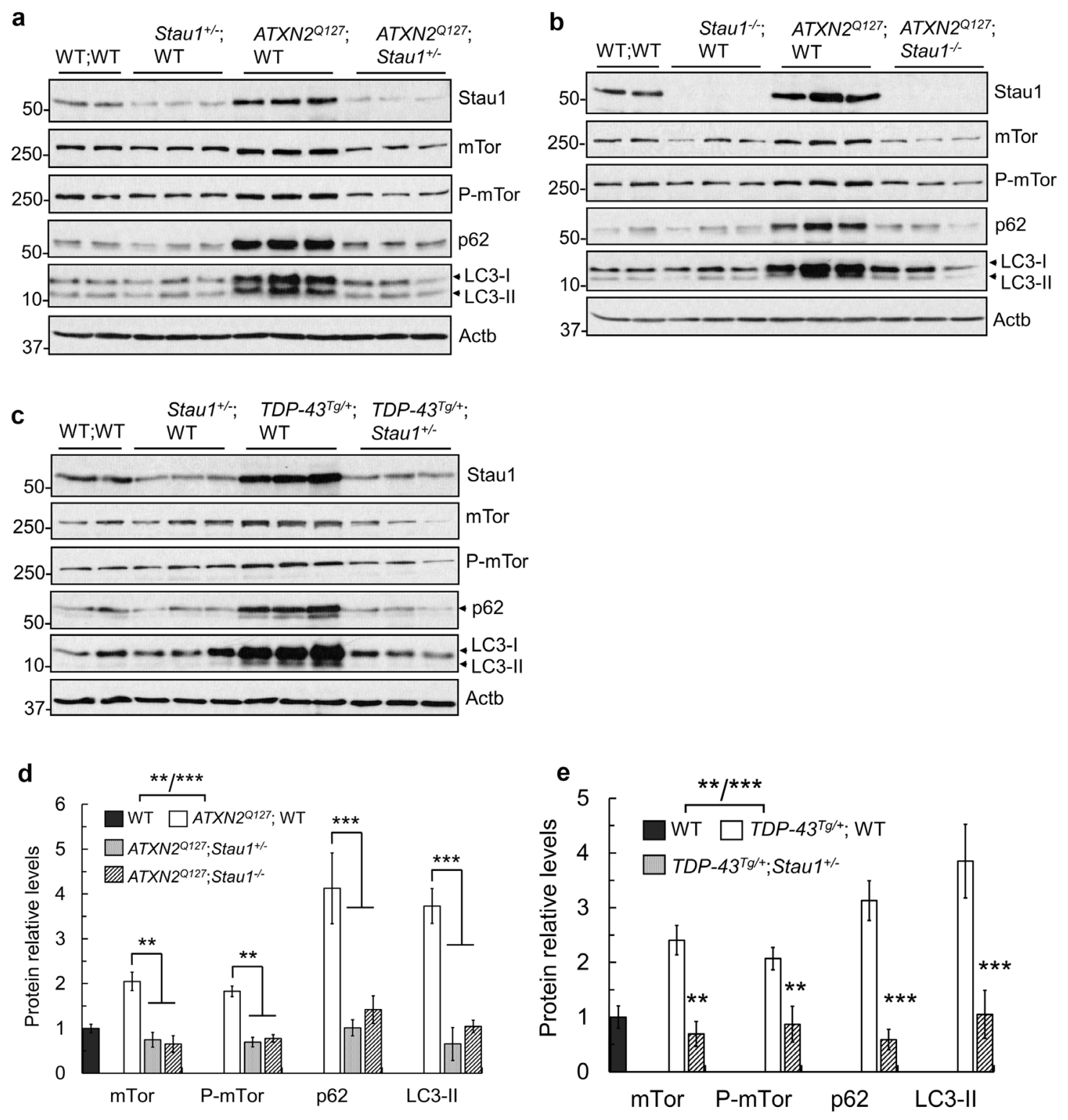

Figure 6 | Reduction of Stau1 levels in vivo attenuates mTor activation in SCA2 and TDP-

43 ALS models. a, b Reduction of Stau1 reduces mTor activity and restores autophagic pathway proteins in $A T X N 2^{Q 127}$ mice. Western blotting of cerebellar extracts from a cross of ATXN2 $2^{Q 127}$ and Stau1//- mice (34 weeks of age) (ref. 3) (a) or ATXN2 ${ }^{\text {Q127 }}$;Stau1 ${ }^{\text {-/ }}$ (20 weeks of age) (b) showing reduced mTor activity and normalization of autophagic pathway proteins in vivo. c Western blotting of spinal cord extracts from $T D P-43^{T g /+}$ hemizygous mice haploinsufficient for Stau1 (22 weeks of age) showing reduced mTor activity and normalization 
of autophagic pathway proteins. Each lane represents extract from an individual mouse. $\beta$-Actin is used as a loading control and the blots are from three replicate experiments. $\mathbf{d}$, e Quantitative analysis of western blots shown in a-c. Data are means $\pm \mathrm{SD},{ }^{* *} P<0.01,{ }^{* * *} P<0.001$, Student t-test 
a

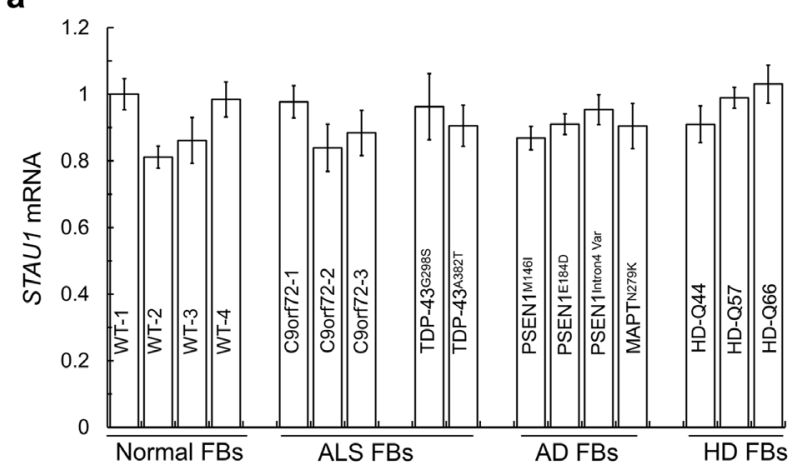

b

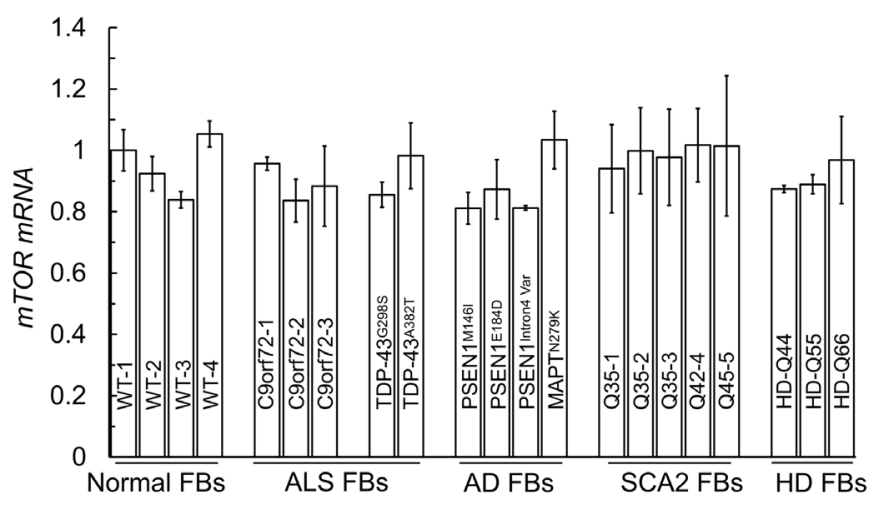

C

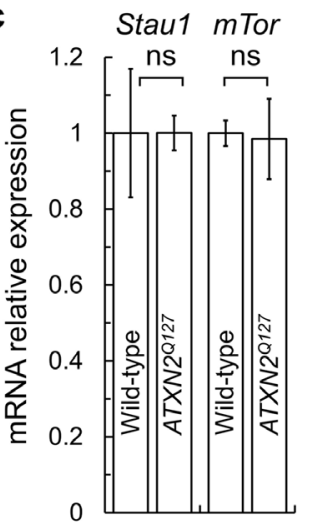

d

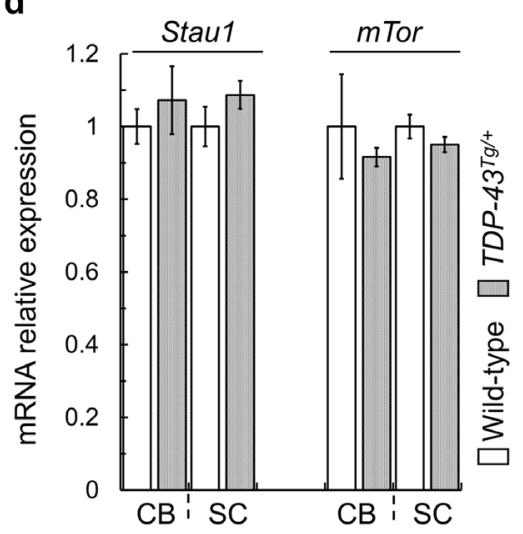

Extended Data Figure 1 | STAU1 and $m$ TOR RNA levels are unaltered in neurodegenerative disease cells and tissues. $\mathbf{a}, \mathbf{b}$ qRT-PCR analyses of ALS, AD, HD and SCA2 FBs revealed unaltered mRNA levels for STAU1 (a) and $m$ TOR (b) compared with control cells. c, d qRT-PCR analyses of Stau1 and mTor mRNAs from cerebellar (CB) and spinal cord (SC) RNAs from ATXN2 ${ }^{Q 127}$ (24 weeks of age; 3 animals per group) and TDP-43 ${ }^{T g / t}$ hemizygous mice ( 8 weeks of age; 3 animals per group) mice compared to wild-type littermates. Gene expression levels were normalized to Actin. 
a

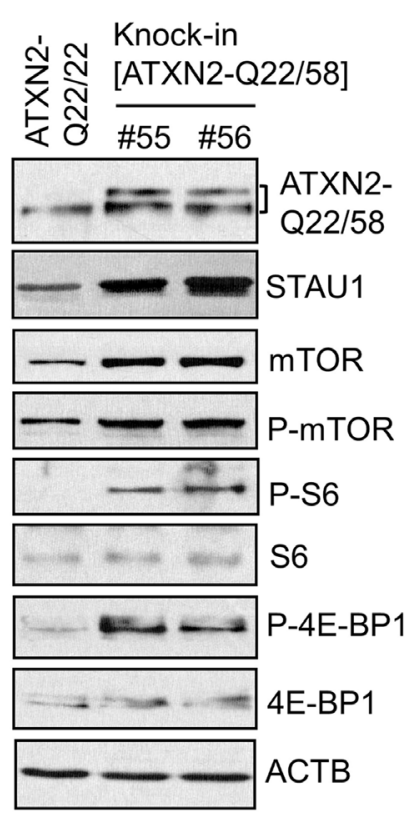

$c$
0
0
0
0
$\frac{0}{0}$
$\frac{0}{4}$
0
$\frac{0}{4}$
$\frac{0}{4}$

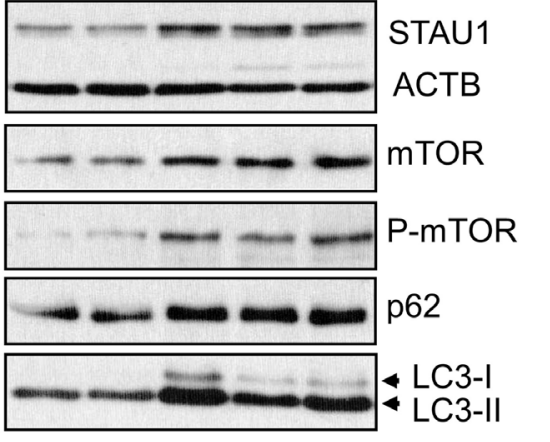

b

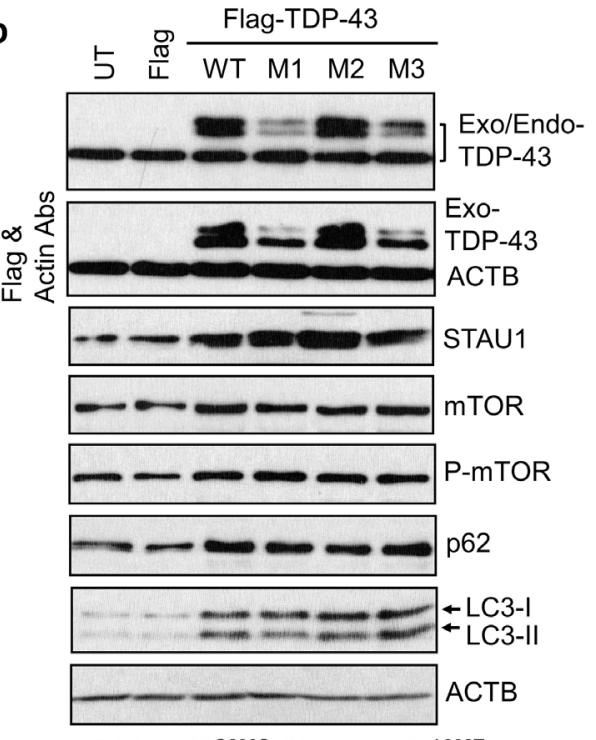

M1: TDP-43' $298 \mathrm{~S} ;$ M2: TDP-43 ${ }^{\text {A382T; }}$ M3: TDP-43G348C

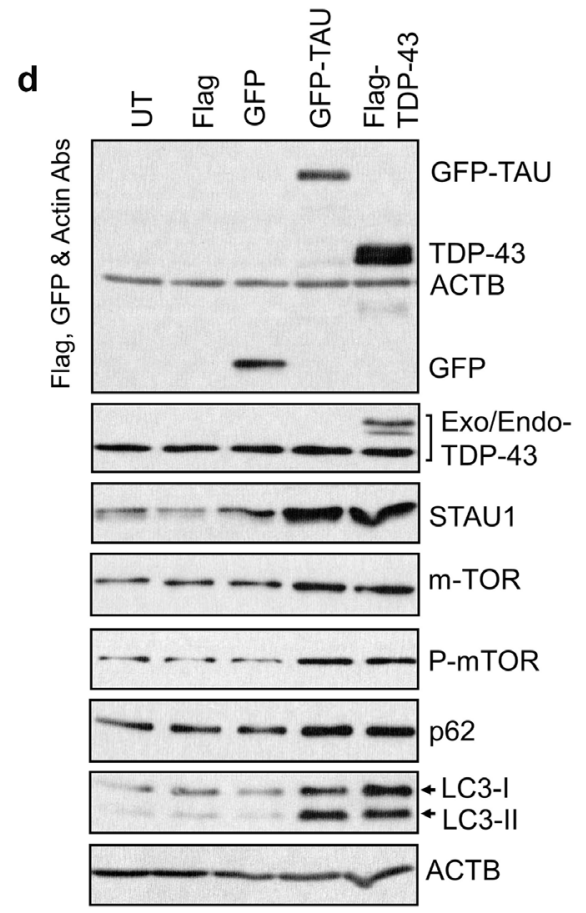

\section{Extended Data Figure 2 | Expression of disease-linked genes recapitulates STAU1} abundances and mTOR activation. a CRISPR/CAS9 edited ATXN2-Q58 KI cells showing increased STAU1, mTOR levels and mTOR activity (increased P-mTOR, P-S6 and P-4E-BP1 levels) on western blot. b-d Exogenous expression of disease-linked genes recapitulates STAU1 abundances and mTOR activation. HEK-293 cells were transfected with the indicated 
plasmid constructs for $48 \mathrm{hr}$ followed by western blotting. Expression of exogenous wild-type or mutant TDP-43 (G298S, A382T, G348C and CTF) (b, c) or TAU (d) resulted in increased STAU1 levels and mTOR activation. $\beta$-Actin was used as loading control and representative blots of three independent experiments are shown 


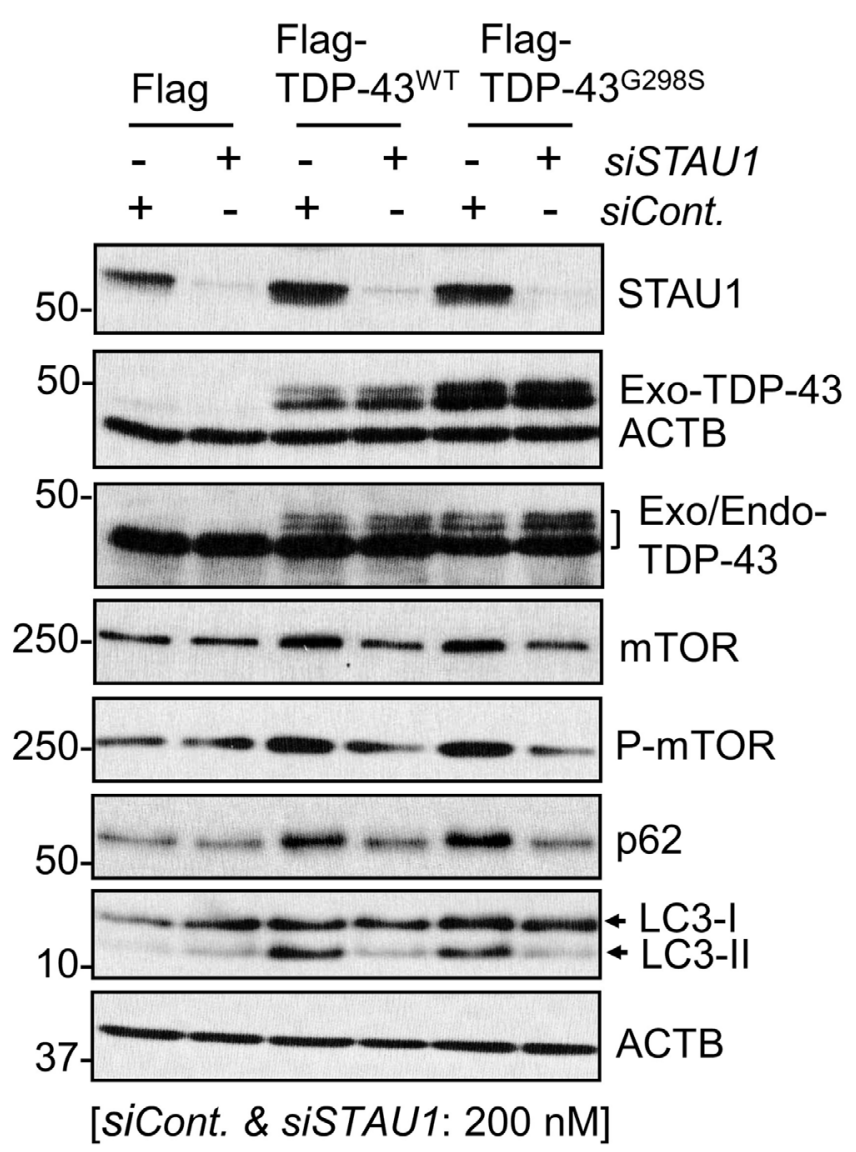

Extended Data Figure 3 | STAU1 depleted cells are resistant to mTOR activation by TDP-

43. HEK-293 cells were treated 72 hrs by STAU1 siRNA and then transfected with Flag-tagged wild-type or mutant TDP-43 for an additional 48 hrs followed by western blotting. STAU1depleted cells have resistance to mTOR activation upon overexpression of disease-linked genes compared with controls. $\beta$-Actin is used as a loading control and the blots are from three replicate experiments. 
Supplementary Table 1. RT-PCR and qRT-PCR primer sequences

\begin{tabular}{llcc} 
GAPDH-F & 5'-ACATCGCTCAGACACCATG-3' & Human & RT-PCR \\
GAPDH-R & 5'-TGTAGTTGAGGTCAATGAAGGG-3' & $"$ & $"$ \\
mTOR-F & 5'-CGAACCTCAGGGCAAGATG-3' & $"$ & $"$ \\
mTOR-R & 5'-TTTCCTCATTCCGGCTCTTTAG-3' & $"$ & $"$ \\
ACTB-F & 5'-GAAAATCTGGCACCACACCT-3' & $"$ & qRT-PCR \\
ACTB-R & 5'-TAGCACAGCCTGGATAGCAA-3' & $"$ \\
mTOR-F & 5'-CAGAAGGTGGAGGTGTTTGAG-3' & $"$ & $"$ \\
mTOR-R & 5'-TGACATGACCGCTAAAGAACG-3' & $"$ & $"$ \\
& & & $"$ \\
Stau1-F & 5'-AGTACATGCTCCTTACAGAACG-3' & Mouse & $"$ \\
Stau1-R & 5'-TGATGCCCAACCTTTACCTG-3' & $"$ & $"$ \\
mTor-F & 5'-ATTCAATCCATAGCCCCGTC-3' & $"$ & $"$ \\
mTor-R & 5'-TGCATCACTCGTTCATCCTG-3' & $"$ & $"$ \\
Actb-F & 5'-CGTCGACAACGGCTCCGGCATG-3' & $"$ & $"$ \\
Actb-R & 5'-GGGCCTCGTCACCCACATAGGAG-3' & $"$ & $"$ \\
\hline
\end{tabular}




\section{Supplementary Information}

\section{Staufen blocks autophagy in neurodegeneration}

Sharan Paul ${ }^{1}$, Warunee Dansithong ${ }^{1}$, Mandi Gandelman ${ }^{1}$, Tao $\mathrm{Zu}^{2}$, Laura P.W. Ranum ${ }^{2}$, Karla P. Figueroa ${ }^{1}$, Daniel R. Scoles ${ }^{1}$, and Stefan M. Pulst ${ }^{1 \#}$

${ }^{1}$ Department of Neurology, University of Utah, 175 North Medical Drive East, 5th Floor, Salt Lake City, Utah, 84132. 'Department of Neurology, University of Florida, Gainesville, FL 32610, USA

\#Author for correspondence: stefan.pulst@hsc.utah.edu

\section{Methods}

\section{DNA constructs}

The plasmid constructs used in this study were 3XFlag-tagged STAU1 or wild-type TDP-43, Histagged STAU1 or GFP (ref. 3) and pRK5-EGFP-TAU (Addgene, Plasmid \#46904). Mutant TDP43 cDNAs (TDP-43 ${ }^{\mathrm{G} 298 \mathrm{~S}}$ and TDP-43 ${ }^{\mathrm{A} 382 \mathrm{~T}}$ ) were PCR-amplified from cDNA libraries of ALS patient FBs with TDP-43 mutations (G298S \#ND32947 and A382T \#ND41003) (Coriell Cell Repositories, Camden, NJ, USA). TDP-43 ${ }^{\mathrm{G} 348 \mathrm{C}}$, C-terminal fragment of TDP-43 (TDP-43 ${ }^{\mathrm{CTF}}$ ) and RNA-binding domain 3 deletion of STAU1 (STAU1[RBDA3]) constructs were generated using TDP-43 and STAU1 cDNAs as templates, respectively ${ }^{3}$. The PCR products were cloned into pCMV-3XFlag plasmid (Agilent Technologies, USA). All constructs were verified by sequencing. $3 X F l a g$ is referred to as Flag in the text and figures.

\section{siRNAs and Reagents}

The siRNAs used in this study were: All Star Negative Control siRNA (Qiagen, Cat\# 1027280) and human siSTAU1: 5'-CCUAUAACUACAACAUGAGdTdT-3' (refs. 3,18). Staufen1 siRNA oligonucleotides were synthesized by Invitrogen, USA. The oligonucleotides were deprotected and the complementary strands were annealed. Thapsigargin (Tocris USA, Cat\# 1138), Tunicamycin (Tocris USA, Cat\# 3516), Ionomycin (Tocris USA, Cat\# 1704) and Sodium arsenite solution (Sigma-Aldrich, Cat\# 1062771000) were used in this study.

\section{Cell culture and Transfections}

Five SCA2 patient-derived skin FBs (ATXN2 with CAG repeats; 35, 35, 35, 42, and 45) were maintained in DMEM medium containing $10 \%$ fetal bovine serum ${ }^{3}$. All subjects gave written 
consent and all procedures were approved by the Institutional Review Board (IRB) at the University of Utah. The following primary human fibroblasts were obtained from the Coriell Cell Repositories (Camden, NJ, USA): Normal FBs (\#ND29510, \#ND34769 and \#ND38530), two ALS patients with TDP-43 mutations (G298S \#ND32947 and A382T \#ND41003), three ALS patients with C9orf72 mutations (\#ND40069, \#ND42504 and \#ND42506), three Huntington disease (HD) patients (Q44 \#ND31038, Q57 \#ND33392 and Q66 \#ND40536) and four Alzheimer's disease (AD) patients [(PSEN1 mutations: M146I \#ND34730, E184D \#ND34732 and Intron4 Var \#ND41001) and MAPT mutation: N279K \#ND40074]. All FBs including HEK293-ATXN2-Q22/58 knock-in (KI) cells ${ }^{3}$ used in this study were maintained in DMEM medium containing $10 \%$ fetal bovine serum.

For over-expression of recombinant proteins, HEK-293 cells were seeded on 6 well dishes and incubated overnight. The cells were then transfected with plasmid DNAs and harvested $48 \mathrm{hrs}$ post-transfection and processed as two aliquots for protein and RNA analyses. For siRNA experiments, cells were transfected with siRNAs using lipofectamine 2000 transfection reagent (ThermoFisher Scientific) according to the manufacturer's protocol. Prior standardization experiments showed that maximum silencing was achieved 4-5 days posttransfection.

\section{Cell line authentication}

We authenticated cell lines used in the study (as HEK-293 cells or human fibroblasts, etc) by short tandem repeat (STR) analysis, an approach that evaluates 24 loci including Amelogenin for sex identification, using the GenePrint 24 System (Promega, USA). We also used PCR sequencing to verify the presence of mutations in patient-derived cells. This is in part in response to $\mathrm{NIH}$ guidelines on scientific rigor in conducting biomedical research (NOT-OD-15103).

\section{Mice}

ATXN2 $^{\text {Q127 }}$ (Pcp2-ATXN2[Q127]) mice ${ }^{22}$ were maintained in a B6D2F1/J background. The Stau1 ${ }^{\text {tm1Apa(--) }}\left(\right.$ Stau1 $\left.^{-/}\right)$mouse ${ }^{35}$ was a generous gift from Prof. Michael A. Kiebler, Ludwig Maximilian University of Munich, Germany, and maintained in a C57BL/6J background. $A T X N 2^{Q 127}$ mice were crossed with Stau1 ${ }^{-/-}$mice to generate $A T X N 2^{Q 127}$;Stau1 ${ }^{+/-}$and $A T X N 2^{W T} ;$ Stau ${ }^{+/-}$mice. These mice were then interbred to generate $A T X N 2^{Q 127} ;$ Stau1 ${ }^{-/-}$and $A T X N 2^{W T}$;Stau1 ${ }^{-/}$mice. These mice were in a mixed background of B6D2F1/J C57BL/6J. 
B6;SJL-Tg(Thy1-TARDBP)4Singh/J (Stock 012836) (ref. 24) purchased from Jackson Laboratories. Jackson Laboratories B6SJLF1/J mice (Stock No. 100012) were backcrossed to C57BL/6J to N2 before crossing them to Stau $1^{-/-}$mouse. Genotyping of animals was accomplished according to published protocols ${ }^{22,24,35}$. All mice were bred and maintained under standard conditions consistent with National Institutes of Health guidelines and conformed to an approved University of Utah IACUC protocol. Wild-type and BAC-C9orf72 mouse ${ }^{27}$ brain extracts were provided by Laura P.W. Ranum, Department of Neurology, University of Florida, Gainesville, FL 32610, USA.

\section{Primary culture of cortical neurons}

Primary cortical neuron cultures were prepared from neonatal wild-type or Stau1/- mice

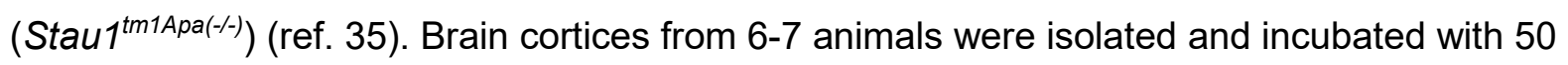
units of papain (Worthington Biochemical, USA) in Earle's balanced salt solution (EBSS) with 1.0 mM L-cysteine and $0.5 \mathrm{mM}$ EDTA for 15 minutes at $37^{\circ} \mathrm{C}$, followed by washing in EBSS and mechanical trituration. The remaining tissues were removed by filtration through a $50 \mu \mathrm{m}$ strainer (Falcon). Neurons were seeded at $50 \times 10^{3}$ per $\mathrm{cm}^{2}$ on plates coated with poly-Lornithine and laminin in Neurobasal Plus medium containing 2\% B27 Plus supplement (Life technologies, USA). On day 2, $10 \mu \mathrm{M}$ cytosine arabinoside was added for $24 \mathrm{hr}$ to prevent proliferation of glial cells and $75 \%$ of culture medium volume was replenished every $2-3$ days from there on. Experiments were conducted on day 9-10 by replacing all culture medium with fresh media containing thapsigargin or vehicle (DMSO), and $18 \mathrm{hr}$ later protein lysates were prepared following standard procedures.

\section{Antibodies}

The antibodies used for western blotting and their dilutions were as follows: mouse anti-Ataxin-2 antibody (Clone 22/Ataxin-2) [(1:4000), BD Biosciences, Cat\# 611378], rabbit anti-Staufen antibody [(1:5000), Novus biologicals, NBP1-33202], LC3B Antibody [(1:8000), Novus biologicals, NB100-2220], TDP-43 antibody [(1:8000), Proteintech, Cat\# 10782-2-AP], monoclonal anti-FLAG M2 antibody [(1:10,000), Sigma-Aldrich, F3165], monoclonal anti- $\beta$ Actin-peroxidase antibody (clone AC-15) [(1:30,000), Sigma-Aldrich, A3854], SQSTM1/p62 antibody [(1:4000), Cell Signaling, Cat\# 5114], mTOR antibody [(1:4000), Cell Signaling, Cat\# 2972], Phospho-mTOR (Ser2448) antibody [(1:3000), Cell Signaling, Cat\# 2971], p70 S6 Kinase antibody [(1:4000), Cell Signaling, Cat\# 9202], Phospho-p70 S6 Kinase (Thr389) antibody 
[(1:3000), Cell Signaling, Cat\# 9205], 4E-BP1 antibody [(1:4000), Cell Signaling, Cat\# 9452], Phospho-4E-BP1 (Thr37/46) (236B4) rabbit mAb [(1:3000), Cell Signaling, Cat\# 2855], 6x-His Tag monoclonal antibody (HIS.H8), HRP [(1:10,000) (ThermoFisher Scientific, MA1-21315HRP)] and sheep-anti-Digoxigenin-POD, Fab fragments [(1:10,000), Roche Life Science, Cat\# 11207733910]. The secondary antibodies were: Peroxidase-conjugated horse anti-mouse IgG $(H+L)$ antibody [(1:5000), Vector laboratories, PI-2000] and Peroxidase-conjugated AffiniPure goat anti-rabbit lgG $(H+L)$ antibody [(1:5000), Jackson ImmunoResearch Laboratories, Cat\# 111-035-144].

\section{Preparation of protein lysates, western blotting and in vitro RNA binding assays}

Cellular extracts were prepared by a single-step lysis method ${ }^{3}$. The harvested cells were suspended in SDS-PAGE sample buffer [Laemmli sample buffer (Bio-Rad, Cat\# 161-0737)] and then boiled for $5 \mathrm{~min}$. Equal amounts of the extracts were used for western blot analyses. Cerebellar or spinal cord protein extracts were prepared by homogenization of mouse tissues in extraction buffer [25 mM Tris-HCl pH 7.6, 300 mM NaCl, 0.5\% Nonidet P-40, 2 mM EDTA, 2 $\mathrm{mM} \mathrm{MgCl} 2,0.5 \mathrm{M}$ urea and protease inhibitors (Sigma-Aldrich, P-8340)] followed by centrifugation at $4^{\circ} \mathrm{C}$ for $20 \mathrm{~min}$ at 14,000 RPM. Only supernatants were used for western blotting. Protein extracts were resolved by SDS-PAGE and transferred to Hybond P membranes (Amersham Bioscience, USA), and then processed for western blotting according to our published protocol ${ }^{3}$. Signals were detected by using the Immobilon Western Chemiluminescent HRP Substrate (EMD Millipore, WBKLSO100) according to the manufacturer's protocol. Blot images were scanned and band intensities were quantified by ImageJ software analyses after inversion of the images, and the relative protein abundances were expressed as ratios to $\beta$ Actin. In vitro RNA binding (Northwestern) assays were performed to determine STAU1 and $m T O R-5$ 'UTR interaction using our previously published protocol ${ }^{3}$.

\section{Immunoprecipitations}

To determine protein-RNA interactions, we carried out protein-RNA immunoprecipitation (IP) experiments using HEK-293 cells expressing Flag-STAU1. The preparation of whole cell extracts and IP methods followed previously published methods ${ }^{3}$. First, cells were lysed with a cytoplasmic extraction buffer [25 mM Tris- $\mathrm{HCl}$ pH 7.6, $10 \mathrm{mM} \mathrm{NaCl}, 0.5 \%$ NP40, 2 mMEDTA, 2 $\mathrm{mM} \mathrm{MgCl}_{2}$, protease inhibitors] and cytoplasmic extracts were separated by centrifugation at 14,000 RPM for $20 \mathrm{~min}$. Second, the resultant pellets were suspended in nuclear lysis buffer or high salt lysis buffer (25 mM Tris- $\mathrm{HCl}, \mathrm{pH} 7.6,500 \mathrm{mM} \mathrm{NaCl}, 0.5 \%$ Nonidet P-40, 2 mM EDTA, $2 \mathrm{mM} \mathrm{MgCl}_{2}$, protease inhibitors), and the nuclear extracts were separated by centrifugation at 
14,000 RPM for $20 \mathrm{~min}$. The nuclear extracts were then combined with the cytoplasmic extracts and denoted whole cell extracts. Specifically, while combining cytoplasmic and nuclear extracts, the $\mathrm{NaCl}$ concentration was adjusted to physiologic buffer conditions ( 150 mM) to preserve in vivo interactions. Ninety percent of cell extracts were subjected to Flag monoclonal antibody (mAb) IP (Anti-FlagM2 Affinity Gel, Sigma-Aldrich, A2220-1ML) to immunoprecipitate STAU1 interacting protein-RNA complexes. The remaining $10 \%$ of whole cell extracts were saved as the input control for western blotting and RT-PCR analyses. The IPs were washed with a buffer containing $200 \mathrm{mM} \mathrm{NaCl}$ and the bound protein-RNA complexes were eluted from the beads with Flag peptide competition (100 $\mathrm{gg} / \mathrm{ml}$ ) (Flag Peptide, Sigma-Aldrich, F3290-4MG). Eluted fractions were divided into two equal parts. One part was analyzed by SDS-PAGE followed by western blotting to determine STAU1 expression. RNA was isolated from the other fraction and subjected to RT-PCR analyses to identify RNAs that bound to STAU1.

\section{RNA expression analyses by quantitative RT-PCR}

Mice were deeply anesthetized with isoflurane then decapitated. Mouse cerebella or spinal cord were removed and immediately submerged in liquid nitrogen. Tissues were kept at $-80^{\circ} \mathrm{C}$ until the time of processing. Total RNA was extracted from mouse tissues and harvested cells using the RNeasy Mini-Kit according to the manufacturer's protocol (Qiagen, USA). DNAse I treated RNAs were used to synthesize cDNAs using the ProtoScript cDNA synthesis kit (New England Biolabs, USA). Quantitative RT-PCR was performed in QuantStudio 12K (Life Technologies, USA) with the Power SYBR Green PCRMaster Mix (Applied Biosystems, USA) using University of Utah genomics core lab. PCR reaction mixtures contained SYBR Green PCRMaster mix, synthesized cDNA and 0.5 pmol primers, and PCR amplifications were carried out for 45 cycles: denaturation at $95^{\circ} \mathrm{C}$ for $10 \mathrm{sec}$, annealing at $60^{\circ} \mathrm{C}$ for $10 \mathrm{sec}$ and extension at $72^{\circ} \mathrm{C}$ for $40 \mathrm{sec}$. The threshold cycle for each sample was chosen from the linear range and converted to a starting quantity by interpolation from a standard curve run on the same plate for each set of primers. Commercial TaqMan assay (Cat\# 4331182: Hs00244999_m1 for STAU1 probe and Hs01060665_g1 for ACTB probe, ThermoFisher Scientific) was used to detect STAU1. All gene expression levels were normalized to $A C T B$ or GAPDH or Actb mRNA levels. Primer pairs designed for RT-PCR and qRT-PCR are given as forward and reverse, respectively, and listed in Supplementary Table 1.

\section{Statistical analysis}


Student's $t$-tests were used to determine whether differences between groups were significant. The level of significance was set at $P \leq 0.05$. ${ }^{*} P \leq 0.05,{ }^{* *} P \leq 0.01,{ }^{* *} P \leq 0.001$ and ns $=P>$ 0.05 . Means \pm SD are presented throughout, unless otherwise specified.

\section{Data availability}

The data are available upon request. 